\title{
Tensión superficial: un modelo experimental con materiales sencillos
}

\author{
Esteban Szigety, Javier Viau, María Alejandra Tintori Ferreira y Horacio Gibbs \\ Universidad Nacional de Mar del Plata, Argentina.grupodidacticadelaciencia@gmail.com
}

[Recibido en junio de 2011, aceptado en marzo de 2012]

En este trabajo se presenta una propuesta didáctica basada en una actividad experimental utilizando materiales de muy bajo costo, orientada a lograr que los alumnos comprendan e interpreten el fenómeno de tensión superficial conjuntamente con la importancia de la modelización en ciencias. Tiene como objetivo principal de enseñanza acercar al alumno a la mecánica de los fluidos estáticos y las fuerzas intermoleculares, combinando contenidos científicos con cuestiones cercanas al estudiante lo que proporciona una motivación adicional a la reflexión de la investigación científica.

Palabras clave: tensión superficial; interacciones moleculares; modelos, experiencias sencillas de físicas.

\section{Superficial tension: experimental model with simple materials}

In this work appears a didactic offer based on an experimental activity using materials of very low cost, orientated to achieving that the student understand and interpret the phenomenon of superficial tension together with the importance of the modeling in sciences. It has as principal aim of education bring the student over to the mechanics of the static fluids and the intermolecular forces, combining scientific contents with questions near to the student what provides an additional motivation to the reflection of the scientific investigation.

Keywords: superficial tension; molecular interactions; models, simple experiences of physical.

\section{Fundamentos teoricos}

\section{Un modelo mecánico de la tensión superficial}

Entre las moléculas de un líquido existen fuerzas intermoleculares de atracción que las mantienen unidas. Una molécula en el seno de un liquido (figura 1) interactúa con las moléculas vecinas por medio de estas fuerzas, permaneciendo en equilibrio a causa de estar rodeada por un gran numero de moléculas, distribuidas homogéneamente en el volumen (Blackwood, Kelly y Bell, 1978).

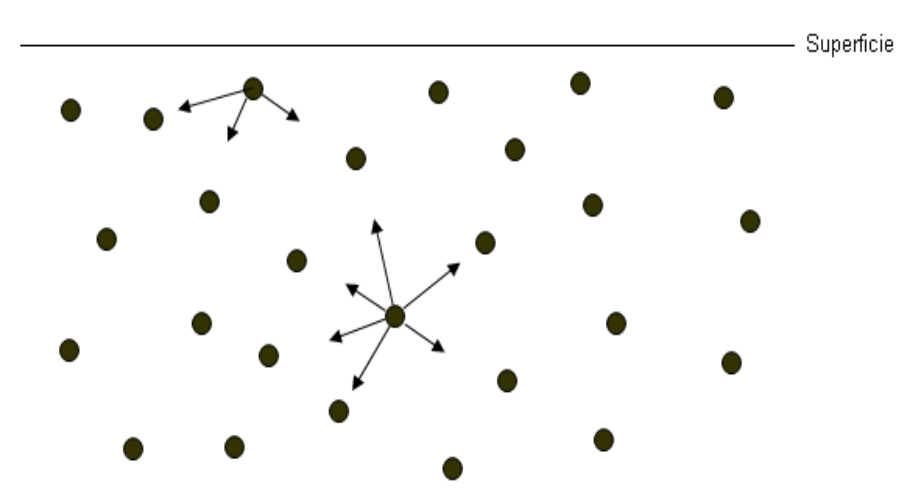

Figura 1. Fuerzas de atracción entre la moléculas de la superficie y las del interior del liquido.
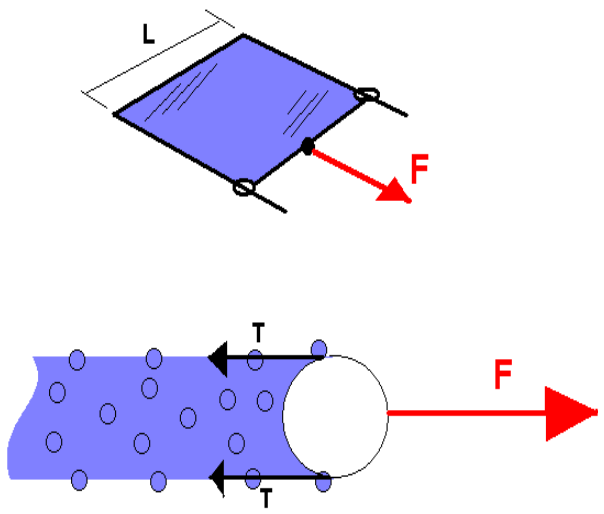

Figura 2. Modelización del fenómeno de tensión superficial.

En la superficie del líquido las fuerzas que atraen a las moléculas hacia abajo no pueden ser anuladas por las moléculas superiores ya que no existen, por lo que la fuerza atractiva neta es 
hacia abajo y perpendicular a la superficie. Sin embargo, las moléculas superficiales se hallan en equilibrio debido a la acción de la fuerza electrostática de repulsión con las moléculas de abajo. En el plano de la superficie, la fuerza neta sobre una molécula es nula, ya que en este plano toda molécula esta siendo tironeada en todas las direcciones, originando la llamada tensión superficial. Este fenómeno es el responsable de que observemos a la superficie del liquido comportase como una especie de membrana elástica.

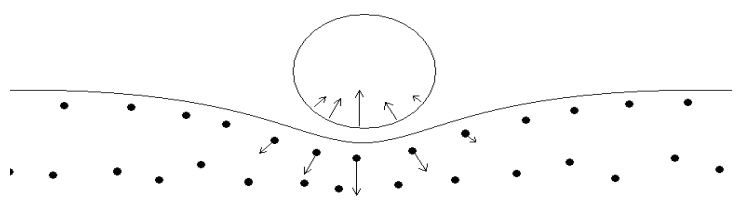

La tensión superficial puede ser cuantificada y evaluada como una magnitud constante para un líquido determinado. Una forma de ponerla en evidencia es por medio de un dispositivo en forma de arco como muestra la figura 2. Si lo sumergimos en un líquido, se observará la

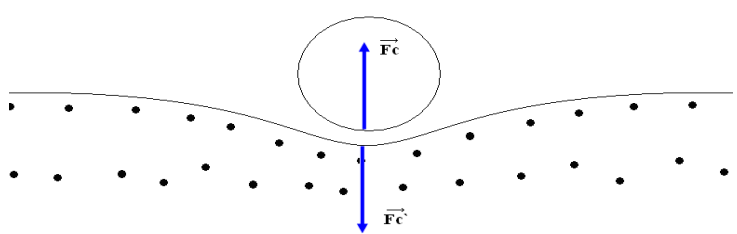

Figura 3. Representación de las fuerzas que se generan en la línea de contacto entre un objeto y la superficie de un liquido. formación de una doble película de líquido sobre el arco, que al estirarla cuasiestáticamente (es decir, muy lentamente) aplicando una fuerza (F) al arco observaremos que esta fuerza resulta ser constante, independientemente del estiramiento $\mathrm{O}$ deformación producida.

Este comportamiento puede resultar contradictorio si se piensa en una membrana de goma o en un resorte, en donde en estos casos la fuerza $(F)$ para estirarlos depende de la deformación. $\mathrm{El}$ hecho de que la fuerza resulte constante se puede explicar en base al modelo molecular ya descripto: al estirar la superficie del liquido y obligar a las moléculas superficiales a separarse, las moléculas que están en el interior de la película van a ser desplazadas a la superficie de forma de ocupar el lugar de la molécula desplazada, permitiendo que la separación de las moléculas superficiales de la superficie se mantenga constante. De esta forma la superficie se podrá extender hasta que el espesor de la película sea tan pequeño que su propio peso la termine por romper. Asimismo, debemos esperar que la fuerza dependa de la longitud L del arco, ya que esta fuerza está desarrollada por unidad de longitud del arco en contacto con el líquido (Mak Wong, 1990). Es por ello que se define al coeficiente de tensión superficial a partir del conocimiento de esta fuerza por unidad de longitud de acuerdo con la siguiente expresión racional:

$$
\mathrm{T}_{\sigma}=\sigma . \mathrm{L}
$$

- Donde $\mathrm{T}_{\sigma}$ es la fuerza de Tensión que ejerce la película sobre el arco.

- L es la longitud del alambre en contacto con el líquido.

- $\sigma$ es la constante de proporcionalidad que se llama comúnmente Tensión Superficial.

En este trabajo se intenta estudiar experimentalmente como la superficie de un líquido es capaz de sostener objetos de mayor densidad que las del líquido. Situaciones cotidianas que se relacionan con este fenómeno son los casos de un insecto caminando sobre la superficie del agua o el de un alfiler flotando sobre la misma. Este fenómeno, donde en apariencia parece violarse el principio de flotabilidad de Arquímedes, se debe a la tensión superficial. Si el peso del objeto es del orden de las fuerzas de tensión superficial, la superficie del líquido lograra sostener al objeto. Pero si perforamos esta capa molecular y el cuerpo entra en contacto con el seno del líquido, por el principio de Arquímedes se termina hundiendo si la densidad del cuerpo supera la del líquido (Gamow, 1969). 
Analicemos con más detalle las fuerzas de repulsión electrostática que actúan sobre un alfiler: cuando colocamos el objeto sobre la superficie del líquido, comienzan a actuar fuerzas entre las moléculas del objeto y las moléculas superficies del líquido (figura 3). Estas fuerzas terminan por generar sobre el alfiler una fuerza de contacto neta vertical hacia arriba, que denominamos $F_{c}$, y una reacción sobre la superficie $F_{c}$ `.

Sobre las moléculas de la superficie (figura 4), actúan las reacciones de estas fuerzas de contacto Fc' (indicada en color azul), que deformaran la superficie. Pero para que las moléculas superficiales alcancen su equilibrio deben estar actuando otras fuerzas: la tensión superficial $\left(\mathrm{T}_{\sigma}\right)$ (en color negro) y la fuerza de repulsión electroestática $\left(\mathrm{F}_{\mathrm{r}}\right)$ (en color rojo) que ejercen las moléculas que están bajo la superficie. En la Figura 4 se muestra un diagrama de fuerzas sobre una porción de la superficie. En este sencillo modelo esquemático para unas pocas moléculas se evidencia que mientras la tensión superficial las mantenga unidas, las moléculas superficiales podrán equilibrar al peso del alfiler.

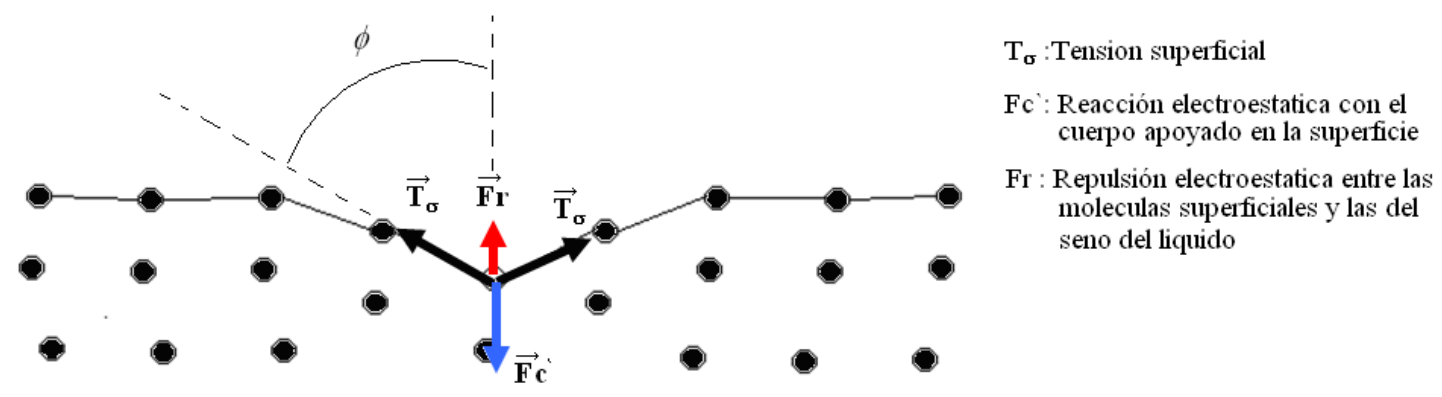

Esta propuesta didáctica está diseñada con el propósito de que los alumnos conceptualicen estas fuerzas y logren cuantificarlas.

Planteando las ecuaciones de equilibrio sobre el alfiler obtenemos:

$$
\mathrm{F}_{\mathrm{c}}=\text { peso del alfiler }=\mathrm{F}_{\mathrm{c}}
$$

y sobre un segmento puntualizado de la superficie (figura 4):

$$
\mathrm{F}_{\mathrm{r}}+2 \cdot \mathrm{T}_{\sigma} \cdot \cos \emptyset=\text { peso del alfiler }
$$

Por más que se desconozca la forma matemática que pueda representar a $F_{r}$, tanto $T_{\square}$ como $F_{r}$ son fuerzas que deben depender de la longitud en contacto con la superficie.

\section{Propuesta didáctica}

\section{Contextualización}

Esta experiencia fue instrumentada en Colegio Dr. Arturo U. Illia, institución que depende de la Universidad Nacional de Mar del Plata, Argentina.

\section{Participantes}

La propuesta fue realizada por alumnos de enseñanza secundaria (16 - 17 años de edad), en la clase de Física durante el desarrollo del tema mecánica de fluidos. 


\section{Objetivos}

Se propuso como objetivo general del trabajo "Despertar el interés en los jóvenes por la Física como ciencia, a partir de la realización de trabajos experimentales de una manera sencilla, práctica e interactiva".

Con respecto a los objetivos de aprendizaje se propuso que los alumnos logren:

- Medir la fuerza de contacto necesaria para romper la superficie de un fluido, en este caso agua de distribución domiciliaria a temperatura ambiente, evaluando su error de medida.

- Estudiar la dependencia de estas fuerzas con la longitud L del objeto en contacto con el fluido.

- Analizar el resultado experimental comparándolo con el modelo mecánico analizado en el marco teórico.

Determinar si el método elegido para romper la superficie del líquido ofrece alguna medida de la tensión superficial del agua.

\section{Marco experimental}

\section{Materiales}

Para llevar a cabo esta experiencia fue necesario contar con los siguientes materiales:

- $1 \mathrm{~m}$ de Alambre fino esmaltado de $0,7 \mathrm{~mm}$ (del que se usa para bobinar motores o transformadores).

- Bandeja que podamos llenar con agua.

- Agua del sistema de distribución domiciliario.

- Pinzas para manipular el alambre.

\section{Procedimientos}

Se deberá construir con el alambre un aro como el mostrado en la figura 5, que tendrá una parte de su longitud $\boldsymbol{y}$ en contacto con el agua y otra parte $\boldsymbol{x}$ fuera del agua. El alambre utilizado para la construcción del aro tiene una longitud total que llamaremos L.

Con el aro se prueba su sustentación sobre la superficie (figura 6), reduciendo paulatinamente la longitud $\boldsymbol{y}$ de modo de alcanzar el límite de rotura, el cual nos estará indicando el punto donde las fuerzas intermoleculares no serán suficientes para sostener el peso del aro.

\section{Orientación didáctica}

Las siguientes actividades y pasos pueden servir de orientación para el desarrollo de la actividad experimental

Actividades previas de discusión y análisis con los alumnos:

- Identificar las variables dependientes, independientes y controladas.

- Analizar los márgenes de error en las mediciones realizadas, ¿tienen el mismo margen de error las variables medidas $\boldsymbol{x}$ e $\boldsymbol{y}$ ?

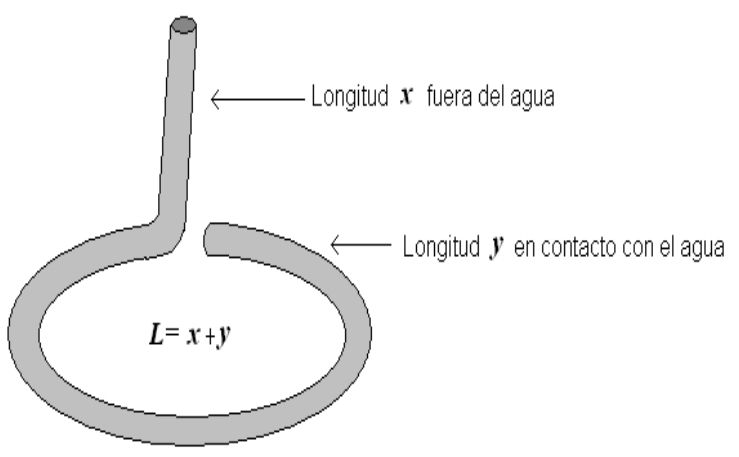

Figura 5. Alambre en forma de aro de longitud $\mathrm{L}=\boldsymbol{x}+\boldsymbol{y}$. 
- Discutir la representación grafica que se espera obtener según el modelo planteado en la ecuación 3 con las variables: L e $\boldsymbol{y}$.

Pasos a seguir en la experiencia:

- Cortar alambres de distinta longitud L.

- Medir con la regla la longitud total L de los alambres cortados.

- Tomar el alambre de menor longitud y darle la forma aproximada de la figura 5, colocándolo con cuidado sobre el agua para observar su equilibrio, si se hunde modificar su forma aumentando la longitud $\boldsymbol{y}$ hasta lograr que flote.

- Disminuir la longitud $\boldsymbol{y}$ del alambre hasta hallar el equilibrio antes de la rotura.

- Medir la longitud $\boldsymbol{x}$ del aro que haya verificado el paso 4)

- Repetir el procedimiento con el resto de los alambres de diferentes longitudes.

\section{Resultados}

\section{Mediciones realizadas}

A los fines de simplificar los cálculos se tomó $\boldsymbol{x}$ como medida directa o dependiente ya que es más fácil de medir una vez que ya se ha doblado el alambre. Luego realizando una simple operación $(\boldsymbol{y}=\mathrm{L}-\boldsymbol{x})$ se obtiene la longitud en contacto con el agua que es en definitiva la variable que interesa representar.

La Tabla 1 muestra algunos de los resultados obtenidos por los alumnos cuando realizaron la experiencia.

Tabla 1. Resultados de las mediciones realizadas.

\begin{tabular}{|c|c|c|}
\hline $\begin{array}{c}\text { Longitud total, } \mathbf{L} \\
(\mathbf{c m}) \\
+/-\mathbf{0 , 2 5} \mathbf{~ c m}\end{array}$ & $\begin{array}{c}\text { Longitud fuera del agua, } \boldsymbol{x} \\
\mathbf{( c m})+/-\mathbf{0 , 2 5}\end{array}$ & $\begin{array}{c}\text { Longitud en contacto con el } \\
\text { agua, } \boldsymbol{y}(\mathbf{c m})+/-\mathbf{0 , 5} \\
\boldsymbol{y}=\mathbf{L}-\mathbf{x}\end{array}$ \\
\hline 7 & 0,5 & 6.5 \\
\hline 9 & 0,6 & 8,4 \\
\hline 12,5 & 1 & 11,5 \\
\hline 14,75 & 1,75 & 13 \\
\hline 17,5 & 2 & 15,5 \\
\hline 21,75 & 2,25 & 19,5 \\
\hline
\end{tabular}

El grafico 1 muestra los resultados de la experiencia al procesar los datos de la tabla 1, con sus rectas de máximas y mínimas obtenidas por la hoja de cálculo Excel.

El resultado experimental para la pendiente promedio es:

$$
m \text { (pendiente })=0,86+/-0,13 \text { (adimensional) }
$$

y para la ordenada al origen es:

$$
\text { b (ordenada al origen): } 0,42+/-1,7 \mathrm{~cm}
$$




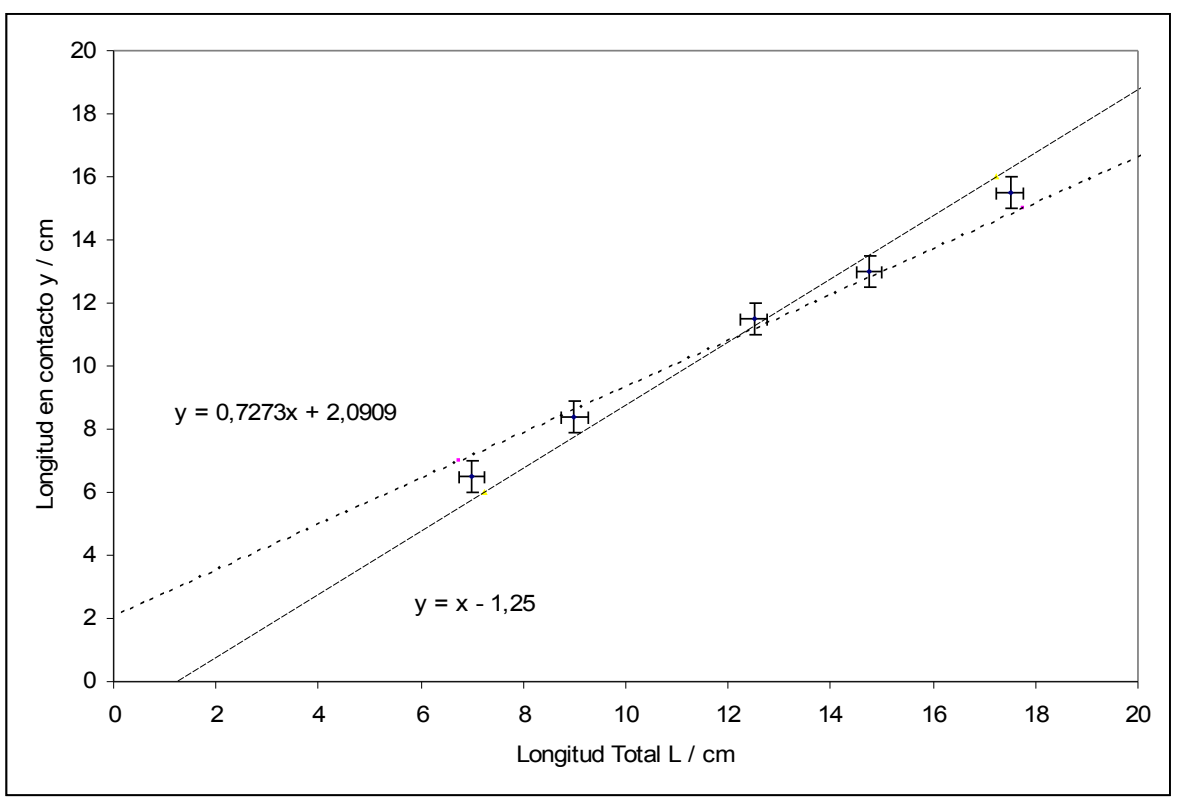

Grafico 1. Representación de longitud de contacto $\boldsymbol{y}$ en función de la longitud total L.

\section{Procesamiento de las mediciones realizadas}

Estos datos son una muestra de los resultados obtenidos en clase. Se observó para otros grupos resultados similares, bajos las mismas condiciones de trabajo: temperatura, muestra de agua de la red y tipo de alambre.

Retomando la ecuación 3 del análisis teórico y aplicándola al aro, tenemos:

$$
\mathrm{F}_{\mathrm{r}}+2 \cdot \boldsymbol{y} \cdot \sigma \cdot \cos \emptyset=\delta \cdot \pi \cdot \mathrm{r}^{2} \cdot \mathrm{L} \cdot \mathrm{g}
$$

En esta expresión, el peso del aro fue reemplazado por, $\mathrm{P}=\mathrm{m} . \mathrm{g}$ donde $\mathrm{m}$ es la masa del alambre, que puede ser expresada como: $\mathrm{m}=\delta . \mathrm{V}$, ( $\varnothing$ es la densidad del material con el que está construido el alambre y $\mathrm{V}$ el volumen del alambre, que es el de un cilindro de longitud $\mathrm{L}$ y radio $\mathrm{r}:\left(\mathrm{V}=\pi \cdot \mathrm{r}^{2} \cdot \mathrm{L}\right)$.

Esta última expresión es la base sobre las que se fundamenta nuestra experiencia a los efectos de estudiar el comportamiento de la superficie del líquido. Las variables controladas son: el radio del alambre $(\mathrm{r})$, la densidad $(\varnothing)$ y la gravedad $(\mathrm{g})$. La variable independiente es el largo total (L). La variable dependiente es la longitud $\boldsymbol{y}$, limite para alcanzar el equilibrio antes de la rotura.

El detalle más importante para resaltar es que todos los grupos obtuvieron una ordenada al origen con un error mayor que el valor obtenido. Esto hace pensar que la recta de mayor ajuste pasa por el origen dentro de los márgenes de error de las medidas. A la vista de este resultado experimental, se confirma lo comentado en el marco teórico, en cuanto a que $\mathrm{F}_{\mathrm{r}}$ depende también de la longitud $\boldsymbol{y}$ en contacto con la superficie del líquido.

Esta conclusión, permite modelizar matemáticamente a $\mathrm{F}_{\mathrm{r}}$ con una relación del tipo:

$$
\mathrm{F}_{\mathrm{r}}=\mathrm{K} \cdot \boldsymbol{y}
$$

Siendo K la constante de proporcionalidad.

De esta manera, la ecuación 4, puede rescribirse de la siguiente forma: 


$$
\boldsymbol{y} \cdot(\mathrm{K}+2 \cdot \sigma \cdot \cos \emptyset)=\pi \cdot \mathrm{r}^{2} \cdot \mathrm{L} \cdot \delta \cdot \mathrm{g}
$$

que muestra matemáticamente una relación lineal entre L e $\boldsymbol{y}$ (con ordenada que pasa por el origen) de la forma

$$
\boldsymbol{y}=m \cdot \mathrm{L}
$$

donde la pendiente

$$
m=\frac{\pi \cdot r^{2} \cdot \delta \cdot g}{K+2 \cdot \sigma \cdot \cos \emptyset}
$$

Algunas de estas constantes son conocidas:

$$
\text { Densidad del cobre: } \delta=8960+/-10 \mathrm{~kg} / \mathrm{m}^{3}
$$

Radio del alambre: $\mathrm{r}=0,35+/-0.01 \mathrm{~mm}=(3,5+/-0,1) \times 10^{-4} \mathrm{~m}$

Gravedad: $\mathrm{g}=9,81+/-0,01 \mathrm{~m} / \mathrm{s}^{2}$

De la pendiente $m$ obtenida en la gráfica y con estas constantes, resulta:

$$
(\mathrm{K}+2 \cdot \sigma \cdot \cos \emptyset)=0,039+/-0,008 \mathrm{~N} / \mathrm{m}
$$

El error calculado fue obtenido sumando los errores relativos de las distintas variables involucradas.

\section{Análisis de los resultados}

El resultado obtenido involucra a la tensión superficial $\sigma$, a la constante $\mathrm{K}$ y al ángulo $\varnothing$. Por lo tanto no se espera que esta experiencia dé como resultado un valor de la tensión superficial para el agua pura a $20^{\circ} \mathrm{C}$ consistente con el valor teórico de los libros de textos que es $0,078 \mathrm{~N} / \mathrm{m}$. Por el contrario la experiencia tiene por objetivo que el alumno mida las fuerzas intermoleculares y contraste los resultados con un modelo teórico.

Se obtuvo en la experiencia una relación lineal entre las variables L e $\boldsymbol{y}$ sin ordenada al origen, confirmando que podemos modelizar para las fuerzas de repulsión electrostática una dependencia con la longitud en contacto con la superficie del líquido.

Si bien no conocemos cual es el ángulo $\varnothing$ que adquiere la superficie antes de la rotura, como la pendiente se verificó constante para todas las mediciones realizadas, podemos estimar que ángulo de ruptura en cada una de ellas ha sido el mismo.
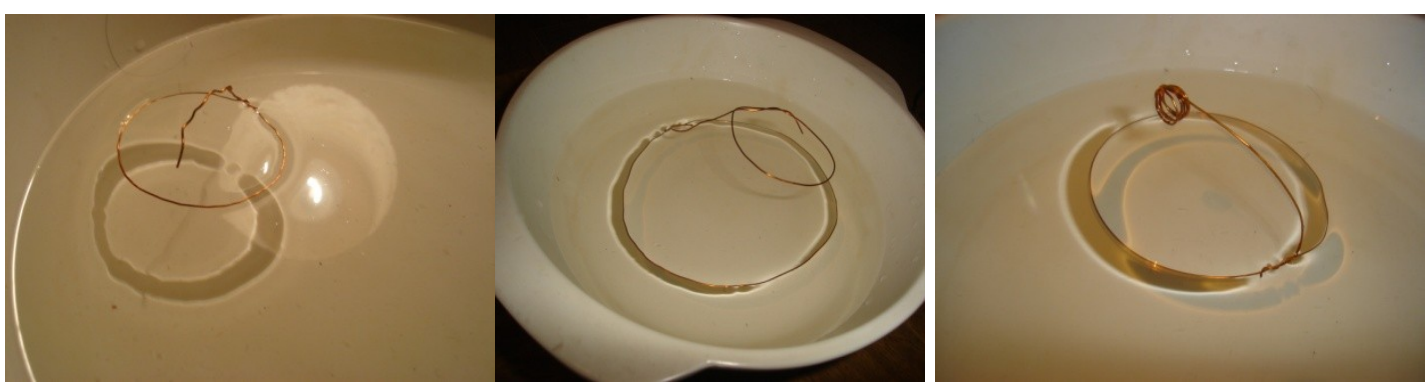

Figura 6. Arreglo experimental para medir la fuerza F.

$\mathrm{Si}$ sugerimos que el valor de $\mathrm{K}$ puede ser despreciable, el menor valor obtenido para el coeficiente $\sigma$, puede explicarse debido al ángulo de ruptura $\varnothing$. Si tomamos la tensión superficial teórica para el agua $(0,078 \mathrm{~N} / \mathrm{m})$, resulta que el ángulo antes de la ruptura formado por la superficie es de $14,5^{\circ}$ con la horizontal $\left(\varnothing=75^{\circ}\right)$.

En la figura 6, las sombras en el fondo del recipiente que fue iluminado con una lámpara de escritorio nos indican la irregularidad del contacto entre el alambre y la superficie, mostrando 
las irregularidades del aro y la distribución de la presión sobre la superficie. Todas estas irregularidades traen como consecuencia errores aleatorios de medida.

\section{Referencias}

Blackwood, O., Kelly W. y Bell R.M. (1978). Fisica general. Editorial CECSA.

George Gamow. (1969). Materia, Tierra y Cielo. Editorial CECSA.

Mak, S.Y. y Wong, K. Y. (1990). The measurement of the surface tension by the method of direct pull. Am. J. Phys. 58 (8), pp. 791-792. 